\title{
Cytotoxic Effect of Post-Purified Herceptin Conjugated Recombinant Diphtheria Toxin on Sk- Br-3 Breast Cancer Cell Line
}

\author{
Sahel Amoozadeh ${ }^{1}$, Mohammad Morad Farajollahi ${ }^{1}$, Maryam \\ Hemmati ${ }^{1}$, Parastoo Tarighi ${ }^{1,}$ \\ ${ }^{1}$ Department of medical biotechnology, Faculty of allied medicine, Iran University of \\ medical sciences, Tehran, Iran \\ * Corresponding author: Parastoo Tarighi, Department of medical biotechnology, \\ Faculty of allied medicine, Iran University of medical sciences, Tehran, Iran. E-mail: \\ tarighi.p@iums.ac.ir
}

DOI: $10.21859 / \mathrm{mci}$-supp-30

Keywords:

Herceptin

Diphtheria Toxin

Sk-Br-3

\begin{abstract}
Introduction: Worldwide, cancer is one the most common causes of death and with its invasive cell growth characteristic, controlling and curing this disease has become more complicated. In breast cancer malignancies, Herceptin (trastuzumab) which is a monoclonal antibody is used as an expensive drug. This cancer medication interferes with the growth of cancer cells which over express the HER2/neu protein by attaching itself to the HER2 receptors on the surface of breast cancer cells and blocking them from receiving growth signals. But the beneficial survival rate of Herceptin is only $20 \%$. Immunotoxin therapies are novel targeted cancer therapies in which a toxin is chemically conjugated to an antibody or growth factor. In this study we introduce an immunotoxin comprising of a truncated diphtheria toxin conjugated to Herceptin antibody in order to improve the effect and potency of the Herceptin drug. We transformed the truncated Diphtheria toxin in an E.coli strain BL21 (DE3). For this reason we eliminated the binding domain sequence of the toxin and expressed these proteins in an expression vector pET28a with the kanamycin resistant gene for selection. The optimization of the Diphtheria toxin expression was due to different Isopropyl $\beta$-D-1-thiogalactopyranoside concentration, induction and sonication time. The toxin was isolated through nickel-sepharose chromatography method. Herceptin and the toxin were conjugated through aldehydes bonding. The effectiveness of this immmunotoxin in inhibiting the proliferation of breast cancer cells was studied on SK-BR-3 cell line.

Conclusions: The cytotoxicity of this immunotoxin on SK-BR-3 cell lines was more effective due to their HER2-neu receptors on the cell surface. The immunotoxin comprising of Diphtheria toxin and herceptin is more effective in killing breast cancer cells with HER2neu receptors in contrast to herceptin alone and has the potential to be used as a therapeutic agent in cancerous cells expressing high levels of HER2-neu receptors.
\end{abstract}

